\section{Dual energy CT pulmonary angiography: findings in a patient with chronic thromboembolic pulmonary hypertension}

A 55-year-old woman became increasingly breathless over a 9month period. Systolic pulmonary artery pressure was estimated at $50 \mathrm{~mm} \mathrm{Hg}$ by echocardiography. CT pulmonary angiography was performed using a dual energy technique whereby datasets are simultaneously acquired at $80 \mathrm{kV}$ and $140 \mathrm{kV}$. Differential absorption of these energy spectra by iodine molecules in intravenous contrast medium is exploited to generate a map of parenchymal perfusion in addition to standard grey-scale images.
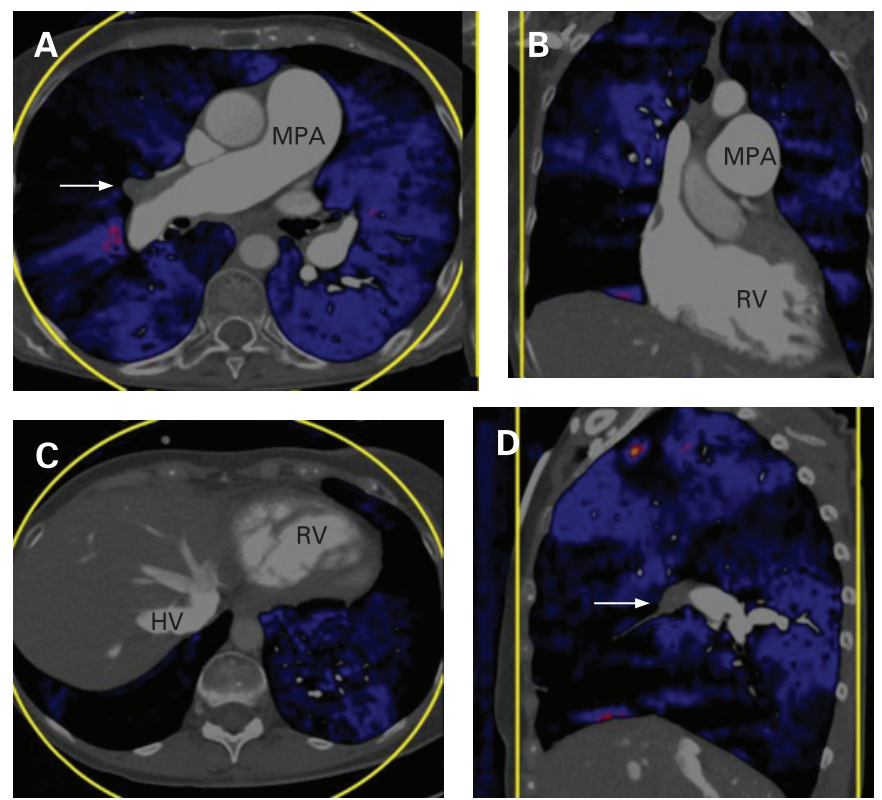

Figure 1 Overlay images from dual energy CT pulmonary angiogram. Areas coloured blue indicate the presence of perfusion and black areas indicate the absence of perfusion. (A) Transaxial image at the level of the main pulmonary artery (MPA) which is dilated, showing occlusion of the origin of the middle lobe artery (arrow) and corresponding wedge-shaped perfusion defect. More subtle areas of reduced perfusion are seen elsewhere. (B) Coronal image through a dilated right ventricle (RV) showing multiple areas of reduced perfusion. (C) Transaxial image at the level of the hepatic veins (HV) which are filled with contrast medium reflecting tricuspid regurgitation. Perfusion defects can be seen at both lung bases. (D) Sagittal image through the right hilum showing occlusion of the middle lobe artery (arrow) and corresponding perfusion defect in the middle lobe. More subtle areas of reduced perfusion can be seen in the right upper and lower lobes.
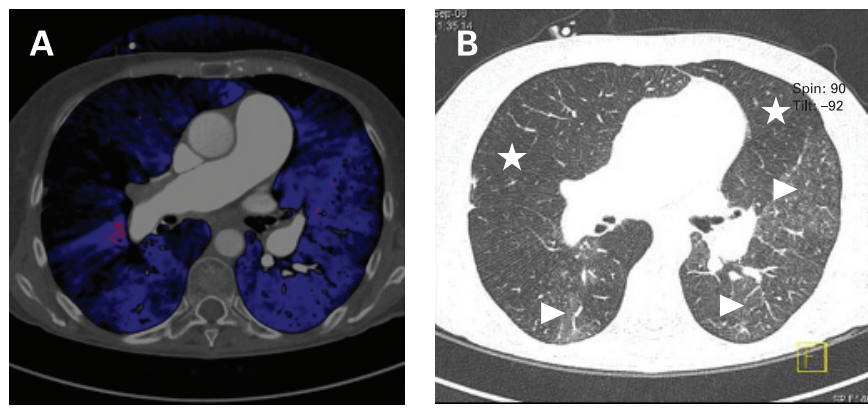

Figure 2 Transaxial images at the level of the main pulmonary artery. (A) Dual energy CT pulmonary angiogram overlay image showing a wedge-shaped perfusion defect in the middle lobe and more subtle areas of reduced perfusion elsewhere. (B) Corresponding grey-scale image on "lung windows" setting (level -600 Hounsfield units (HU), width $1600 \mathrm{HU}$ ) showing a mosaic perfusion pattern with reduced attenuation and vessel size in the middle and left upper lobe (stars) and increased attenuation with larger vessels in the right lower lobe, lingula and left lower lobe (arrowheads).

\section{Learning points}

- Chronic pulmonary thromboembolism with heavy and proxima disease burden is potentially curable with thromboendarterectomy surgery.

- Dual energy CT pulmonary angiography is a novel technique that provides both functional and anatomical information in a single test.

Multiple laminated thrombi and web stenoses were demonstrated and their functional significance elegantly shown by corresponding perfusion defects on the dual energy perfusion images (figs 1 and 2). Pulmonary thromboendarterectomy was performed and recovery was uneventful.

\section{E T D Hoey, S K B Agrawal, V Ganesh, D Gopalan, N J Screaton}

Papworth Hospital, Cambridge, UK

Correspondence to: Dr E T D Hoey, Diagnostic Centre, Papworth Hospital, Papworth Everard, Cambridgeshire CB23 3RE, UK; edwardhoey1@gmail.com

Competing interests: None.

Patient consent: Obtained.

Provenance and peer review: Not commissioned; externally peer reviewed.

Accepted 25 February 2009

Thorax 2009;64:1012. doi:10.1136/thx.2008.112128

\section{REFERENCE}

1. Johnson TR, Krauss B, Sedlmair M, et al. Material differentiation by dual energy CT: initial experience. Eur Radiol 2007;17:1510-7. 